\title{
Faster Correlation Attack on Bluetooth Keystream Generator E0
}

\author{
Yi Lu* and Serge Vaudenay \\ EPFL \\ http://lasecwww.epfl.ch
}

\begin{abstract}
We study both distinguishing and key-recovery attacks against E0, the keystream generator used in Bluetooth by means of correlation. First, a powerful computation method of correlations is formulated by a recursive expression, which makes it easier to calculate correlations of the finite state machine output sequences up to 26 bits for E0 and allows us to verify the two known correlations to be the largest for the first time. Second, we apply the concept of convolution to the analysis of the distinguisher based on all correlations, and propose an efficient distinguisher due to the linear dependency of the largest correlations. Last, we propose a novel maximum likelihood decoding algorithm based on fast Walsh transform to recover the closest codeword for any linear code of dimension $L$ and length $n$. It requires time $O\left(n+L \cdot 2^{L}\right)$ and memory $\min \left(n, 2^{L}\right)$. This can speed up many attacks such as fast correlation attacks. We apply it to E0, and our best key-recovery attack works in $2^{39}$ time given $2^{39}$ consecutive bits after $O\left(2^{37}\right)$ precomputation. This is the best known attack against E0 so far.
\end{abstract}

\section{Background}

Correlation properties play an important role in the security of nonlinear LFSRbased combination generators in stream ciphers. As name implies, the word correlation in stream ciphers is frequently referred to as the intrinsic relation between the keystream and a subset of the LFSR subsequences. The earliest studies dated back to $[21,25,27]$ in the 80 's and the concept of correlation immunity was proposed as a security criterion. In the 90's Meier-Staffelbach [22] analyzed correlation properties of combiners with one memory bit, followed by Golić [12] focusing on correlation properties of a general combiner with $m$-bit memory. Recently, a series of fast correlation attacks sprang up, to name but a few $[5-7,16,24]$. Thereupon we dedicate this paper to the generalized correlation attacks against E0, a combiner with 4-bit memory used in the short-range wireless technology Bluetooth. Prior to our work, existed various attacks $[1,8,10,11$, 13-15, 17, 26] against E0. The best key-recovery attacks are algebraic attacks $[1$,

\footnotetext{
* Supported in part by the National Competence Center in Research on Mobile Information and Communication Systems (NCCR-MICS), a center of the Swiss National Science Foundation under the grant number 5005-67322.
} 
8], whose basic approach is to use the polynomial canceling all memory bits and involving only key bits, instead of considering the multiple polynomial to cancel the key bits in the distinguishing attack; besides, $[9,13,14]$ discussed correlations of E0. In [14], Hermelin-Nyberg for the first time presented a rough computation method to compute the correlation (called bias for our purpose), but neither did they formalize the computation systematically, nor did they attempt to find a larger correlation. In $[9,13]$, two larger correlations for a short sequence of up to 6 bits were exposed. However, due to the limit of the computation method, no one was certain about the existence of a larger correlation for a longer sequence, which is critical to the security of E0.

Our first contribution in the paper is that based on Hermelin-Nyberg [14] we formulate a powerful computation method of correlations by a recursive expression, which makes it easier to calculate correlations of the Finite State Machine (FSM) output sequences up to 26 bits for E0 (and allows us to prove the two known correlations to be the only largest for the first time). Second, we apply the concept of convolution to the analysis of the distinguisher based on all correlations, which allows us to build an efficient distinguisher that halves the data complexity of the basic uni-bias-based distinguisher due to the linear dependency of the two largest biases. Our best distinguishing attack takes $2^{43}$ time given $2^{43}$-bit keystream with $O\left(2^{45}\right)$ precomputation ${ }^{1}$. Finally, by means of Fast Walsh Transform (FWT), we propose a novel Maximum Likelihood Decoding (MLD) algorithm to recover the closest codeword for any linear code. Our proposed algorithm can be easily applied to speed up a class of fast correlation attacks. Furthermore the algorithm is optimal when the length $n$ of the code and the dimension $L$ satisfy the relation $n \geq 2^{L}$, which is the case when we apply it to recover $R_{1}$ for E0. Our best key-recovery attack works in $2^{39}$ time given $2^{39}$ consecutive bits after $O\left(2^{37}\right)$ precomputation. Compared with the minimum time complexity $O\left(2^{49}\right)$ in algebraic attacks $[1,8]$, this is the best known attack against E0.

This paper is structured as follows: in Section 2, a description of E0 is given. In Section 3, we analyze the bias inside E0 systematically. Then based on one largest bias, we build a primary distinguisher for E0 in Section 4; an efficient way is shown in Section 5 that makes full use of all the largest biases to advance the distinguisher. In Section 6 we investigate the MLD algorithm for a linear code; the result is then applied to a key-recovery attack against E0 in Section 7. Finally we conclude in Section 8.

\section{Description of the Bluetooth Keystream Generator E0}

As specified in [3], the keystream generator E0 used in Bluetooth belongs to a combination generator with four memory bits $^{2}$, denoted by $\sigma_{t}=\left(c_{t-1}, c_{t}\right)$ at time $t$, where $c_{t}=\left(c_{t}^{1}, c_{t}^{0}\right)$. The whole system (Fig.1) uses four Linear Feedback

\footnotetext{
${ }^{1}$ Throughout this paper, $O(\cdot)$ is used to provide a rough estimate on complexities, eg. $O\left(2^{45}\right)$ here means $c \cdot 2^{45}$ operations, where $c$ is a small constant.

2 The description of E0 (sometimes called one-level E0) here only involves the keystream generation after the initialization.
} 


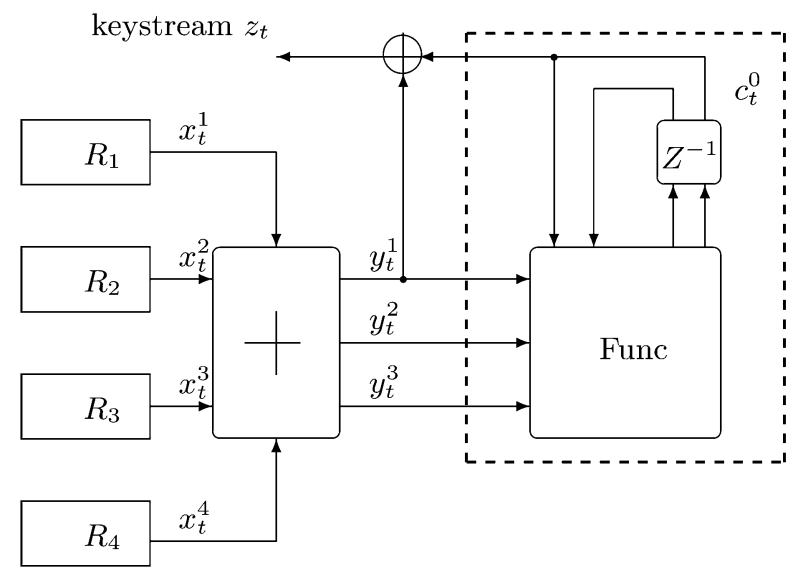

Fig. 1. Outline of E0

Shift Registers (LFSRs) denoted by $R_{1}, \ldots, R_{4}$ with lengths $L_{1}=25, L_{2}=31$, $L_{3}=33, L_{4}=39$ and primitive feedback polynomials

$$
\begin{aligned}
& p_{1}(x)=x^{25}+x^{20}+x^{12}+x^{8}+1, \\
& p_{2}(x)=x^{31}+x^{24}+x^{16}+x^{12}+1, \\
& p_{3}(x)=x^{33}+x^{28}+x^{24}+x^{4}+1, \\
& p_{4}(x)=x^{39}+x^{36}+x^{28}+x^{4}+1,
\end{aligned}
$$

respectively. At clock cycle $t$, the four LFSRs' output bits $x_{t}^{i}, i=1, \ldots, 4$, will be added as integers. The sum $y_{t} \in\{0, \ldots, 4\}$ is represented in the binary system. Let $y_{t}^{i}$ denote its $i$-th least significant bit $(i=1,2,3)$. A 16-state machine (the dashed box in Fig.1) emits one bit $c_{t}^{0}$ out of its state $\sigma_{t}=\left(c_{t-1}, c_{t}\right)$ and takes the input $y_{t}$ to update $\sigma_{t}$ by $\sigma_{t+1}$. Finally, the keystream $z_{t}$ is obtained by xoring $y_{t}^{1}$ with $c_{t}^{0}$. That is,

$$
x_{t}^{1} \oplus x_{t}^{2} \oplus x_{t}^{3} \oplus x_{t}^{4} \oplus c_{t}^{0}=z_{t} .
$$

The detailed mechanism of the FSM is beyond the scope of the paper except the fact that the embedded delay cell (the box labeled $Z^{-1}$ in Fig.1) makes $c_{t}^{0}$ depend only on the initial state $\sigma_{0}$ of the FSM as well as the past vectors $y_{t-1}, y_{t-2}, \ldots, y_{0}$. For completeness, we briefly outline it: given $y_{t}$ together with the state $\sigma_{t}$, the FSM moves into the state $\sigma_{t+1}$. Table 1 shows the state transition of the FSM, where the four-bit state is represented in the quaternary system (e.g. the FSM changes from $\sigma_{t}=13$ into $\sigma_{t+1}=32$ by the input $y_{t}=2$ ).

More formally, by introducing two temporary bits $s_{t+1}=\left(s_{t+1}^{1}, s_{t+1}^{0}\right)$ each clock, the following indirect iterative expressions between $s_{t+1}$ and $c_{t+1}$ suffice to update $c_{t}$ :

$$
\begin{aligned}
& s_{t+1}=\left\lfloor\frac{y_{t}+2 \cdot c_{t}^{1}+c_{t}^{0}}{2}\right\rfloor, \\
& c_{t+1}^{1}=s_{t+1}^{1} \oplus c_{t}^{1} \oplus c_{t-1}^{0},
\end{aligned}
$$


Table 1. State transition of $\sigma_{t+1}$ given $y_{t}$ and $\sigma_{t}$

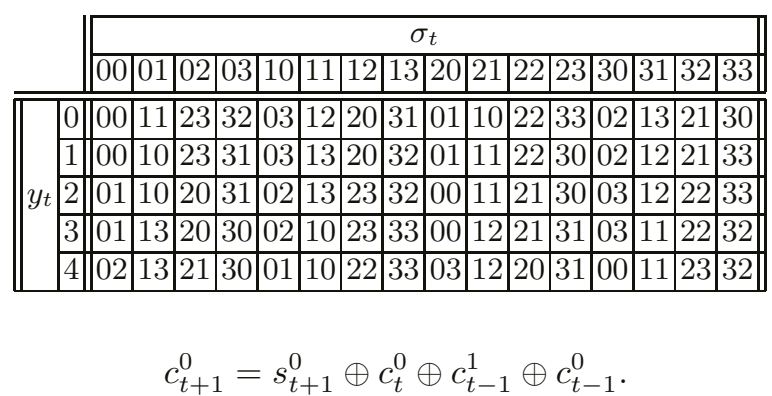

One can check Table 1 by those equations. We denote $\lambda_{t}$ hereafter the content of LFSRs at time $t$. Then the state of E0 at time $t$ is fully represented by the pair $\left(\lambda_{t}, \sigma_{t}\right)$.

\section{Biases Inside E0}

Property 1. Assuming $y_{t}=2$ holds for $t=t_{0}, t_{0}+1, \ldots, t_{0}+3$, then

$$
c_{t_{0}}^{0} \oplus c_{t_{0}+1}^{0} \oplus c_{t_{0}+2}^{0} \oplus c_{t_{0}+3}^{0} \oplus c_{t_{0}+4}^{0}=1 .
$$

Proof. It's easy to verify that the state transition given $y_{t}=2$ (the third bottom row in Table 1) is indeed a linear transformation over $G F(2)^{4}$, that actually satisfies the recurrence relation: $\sigma_{t+1}=A \times \sigma_{t} \oplus 01$, where states $\sigma_{t+1}$ are represented by column vectors of $\left(c_{t}^{1}, c_{t}^{0}, c_{t+1}^{1}, c_{t+1}^{0}\right)$, and $A$ is the following $4 \times 4$ square matrix over $G F(2)$ :

$$
A=\left(\begin{array}{llll}
0 & 0 & 1 & 0 \\
0 & 0 & 0 & 1 \\
0 & 1 & 0 & 0 \\
1 & 1 & 1 & 1
\end{array}\right)
$$

Note that $x^{4}+x^{3}+x^{2}+x+1$ is the minimal polynomial of $A$, from which we deduce $\sigma_{t_{0}} \oplus \sigma_{t_{0}+1} \oplus \sigma_{t_{0}+2} \oplus \sigma_{t_{0}+3} \oplus \sigma_{t_{0}+4}=33$.

Remark 2. Since $\operatorname{Pr}\left(y_{t}=2\right)=\frac{6}{16}$, this seemingly suggests that

$$
\operatorname{Pr}\left(c_{t}^{0} \oplus c_{t+1}^{0} \oplus c_{t+2}^{0} \oplus c_{t+3}^{0} \oplus c_{t+4}^{0}=1\right) \approx \frac{1}{2}+\left(\frac{6}{16}\right)^{4}=\frac{1}{2}+\frac{81}{4096} .
$$

As mentioned in $[9,13]$ (without relating to the above special case), this bit exhibits a much higher bias as shown later in Corollary 7 . We will now introduce essential material in order to find a systematic algorithm to compute biases.

Proposition 3. If $\left(\lambda_{0}, \sigma_{0}\right)$ is random and uniformly distributed, then for any $t$

- $\left(\lambda_{t}, \sigma_{t}\right)$ is random and uniformly distributed,

$-\left(c_{t}, c_{t-1}, \ldots, c_{t-24}\right)$ is independent of $y_{t}$. 
Proof (sketch). The former half of the theorem is justified by the fact that $\left(\lambda_{t}, \sigma_{t}\right)$ is a permutation of $\left(\lambda_{0}, \sigma_{0}\right)$ for any $t$. About the latter half of theorem, first, we know that $\left(\lambda_{t-24}, \sigma_{t-24}\right)$ is random and uniformly distributed by previous conclusion. Thus, $y_{t-24}, \ldots, y_{t-1}$ are i.i.d. random variables all independent of both $\sigma_{t-24}$ and $y_{t}$. By Eq. $(2,3,4)$, we complete the proof.

Interestingly, we deduce that if $\left(\lambda_{0}, \sigma_{0}\right)$ is uniformly distributed, then any sequence of 39-bit consecutive E0 keystream is uniformly distributed; in particular, no better key-recovery attack against E0 exists other than tradeoffs given a sequence of 39-bit consecutive keystream.

The following definition is derived from normalized correlation [22, p.71].

Definition 4. The bias of a random Boolean variable $X$ is defined as

$$
\Delta(X)=\operatorname{Pr}(X=0)-\operatorname{Pr}(X=1)=E\left[(-1)^{X}\right] .
$$

The normalized correlation between two random Boolean variables $X$ and $Y$ is just the bias of $X \oplus Y$. Assuming that $y_{t}$ is the sum of four balanced independent random bits and that $c_{t}$ is uniformly distributed, then we know that $\Delta\left(a \cdot s_{t+1} \oplus w \cdot c_{t}\right)$ is a constant for any $a, w \in G F(2)^{2}$, denoted by $\Omega(a, w)$. Table 2 shows $\Omega(a, w)$ computed by Eq.(2), where dashed entries are zeros. The following important lemma (see Appendix A for proof) inspired by [14], gives an easy way of computing the bias for iterative structures.

Table 2. Bias of all linear combination of $s_{t+1}$ and $c_{t}: \Omega(a, w)$

\begin{tabular}{||c|c||c|c|c|c||}
\hline \multicolumn{2}{|c||}{$\Omega(a, w)$} & \multicolumn{4}{c||}{$w$} \\
\cline { 2 - 6 } & 0 & 1 & 2 & 3 \\
\hline \hline \multirow{3}{*}{$a$} & 0 & - & - & - & - \\
\cline { 2 - 6 } & 1 & - & - & $-1 / 4$ & - \\
\cline { 2 - 6 } & 2 & - & $1 / 4$ & $5 / 8$ & - \\
\cline { 2 - 6 } & 3 & $-5 / 8$ & - & - & $1 / 4$ \\
\hline
\end{tabular}

Lemma 5. Given $f: \mathcal{E} \times G F(2)^{k} \rightarrow G F(2)$ and $g: G F(2)^{m} \rightarrow G F(2)^{k}$, let $X$ and $Y$ be two independent random variables in $\mathcal{E}$ and $G F(2)^{m}$ respectively. Assuming that $g(Y)$ is uniformly distributed in $G F(2)^{k}$, for any $v \in G F(2)^{m}$, we have

$\Delta(f(X, g(Y)) \oplus v \cdot Y)=\sum_{w \in G F(2)^{k}} \Delta(f(X, g(Y)) \oplus w \cdot g(Y)) \cdot \Delta(w \cdot g(Y) \oplus v \cdot Y)$.

Corollary 6. We set $h:\left(x^{1}, x^{0}\right) \mapsto\left(x^{0}, x^{1} \oplus x^{0}\right)$ to be a permutation defined over $G F(2)^{2}$, and $\delta\left(a_{1}, \ldots, a_{d}\right)=\Delta\left(a_{1} \cdot c_{1} \oplus \cdots \oplus a_{d} \cdot c_{d}\right)$, where $a_{1}, \ldots, a_{d} \in$ $G F(2)^{2}$. Assuming $\left(\lambda_{0}, \sigma_{0}\right)$ is uniformly distributed, for any $d \leq 26$, we have

$$
\delta\left(a_{1}, \ldots, a_{d}\right)=\sum_{w \in G F(2)^{2}} \Omega\left(a_{d}, w\right) \cdot \delta\left(a_{1}, \ldots, a_{d-3}, a_{d-2} \oplus h\left(a_{d}\right), a_{d-1} \oplus a_{d} \oplus w\right) .
$$


Proof. By Eq. $(2,3,4)$ we have

$\delta\left(a_{1}, \ldots, a_{d}\right)=\Delta\left(a_{d} \cdot s_{d} \oplus a_{1} \cdot c_{1} \oplus \cdots \oplus\left(a_{d-2} \oplus h\left(a_{d}\right)\right) \cdot c_{d-2} \oplus\left(a_{d-1} \oplus a_{d}\right) \cdot c_{d-1}\right)$.

Then we apply Lemma 5 with $X=y_{d-1}, Y=\left(c_{1}, \ldots, c_{d-1}\right), g(Y)=c_{d-1}$, $f(X, g(Y))=a_{d} \cdot s_{d}$ and $v=\left(a_{1}, \ldots, a_{d-3}, a_{d-2} \oplus h\left(a_{d}\right), a_{d-1} \oplus a_{d}\right)$, and we obtain

$$
\delta\left(a_{1}, \ldots, a_{d}\right)=\sum_{w} \Omega\left(a_{d}, w\right) \delta\left(a_{1}, \ldots, a_{d-3}, a_{d-2} \oplus h\left(a_{d}\right), a_{d-1} \oplus a_{d} \oplus w\right) .
$$

Note that the assumption of Lemma 5 holds by Proposition 3 .

Now we use Corollary 6 iteratively to deduce some important biases of $\left\{c_{t}^{0}\right\}$ with Table 2 and the initial values $\delta(0,0)=1$, and $\delta(a, b)=0$ for $(a, b) \neq(0,0)$. A full list of nonzero triplets is given below for illustration:

$$
\begin{array}{lll}
\delta(0,0,0)=1, & \delta(1,3,2)=\frac{1}{4}, & \delta(2,3,3)=-\frac{5}{8}, \\
\delta(1,0,2)=\frac{5}{8}, & \delta(2,0,3)=\frac{1}{4}, & \delta(3,3,1)=-\frac{1}{4} .
\end{array}
$$

Corollary 7. Assuming $\left(\lambda_{0}, \sigma_{0}\right)$ is random and uniformly distributed, we have

$$
\begin{aligned}
\operatorname{Pr}\left(c_{t}^{0} \oplus c_{t+1}^{0} \oplus c_{t+2}^{0} \oplus c_{t+3}^{0} \oplus c_{t+4}^{0}=1\right) & =\frac{1}{2}+\frac{25}{512}, \\
\operatorname{Pr}\left(c_{t}^{0}=c_{t+5}^{0}\right) & =\frac{1}{2}+\frac{25}{512} .
\end{aligned}
$$

Note that both biases were mentioned in $[9,13]$ (without formal proof). Now by Corollary 6 , we can easily prove it as shown next.

Proof. We show the equivalent $\delta(1,1,1,1,1)=-\frac{25}{256}$ of the first bias as follows:

$$
\begin{aligned}
\delta(1,1,1,1,1) & =\sum_{w} \Omega(1, w) \cdot \delta(1,1,2, w) \\
& =-\frac{1}{4} \delta(1,1,2,2) \\
& =-\frac{1}{4} \sum_{w} \Omega(2, w) \cdot \delta(1,0, w) \\
& =-\frac{1}{4} \times\left(\frac{1}{4} \delta(1,0,1)+\frac{5}{8} \delta(1,0,2)\right) \\
& =-\frac{25}{256} .
\end{aligned}
$$

The second bias is similarly proved from $\delta(1,0,0,0,0,1)=\frac{25}{256}$.

Also, we computed all $\ell$-tuple biases for $\ell \leq 26$ and found that $\delta(1,1,1,1,1)$, $\delta(1,0,0,0,0,1)$ are the only largest ones. All biases for $\ell=6$ are listed in Table 14, Appendix C. Throughout the paper we let

$$
\gamma=\delta(1,0,0,0,0,1)=-\delta(1,1,1,1,1)=\frac{25}{256}
$$




\section{A Primary Distinguisher for E0}

\subsection{The Connection Polynomial of the Equivalent Single LFSR}

Let $\theta_{i}$ be the order of the connection polynomial $p_{i}(x)$ of $R_{i}$, for $i=1,2,3,4$. Since all $p_{i}(x)$ are primitive polynomials, $\theta_{i}=2^{L_{i}}-1$; furthermore, by Lemma 6.57 of $[18$, p.218], the equivalent LFSR to generate the same sequence of the sum of the four original LFSRs outputs over $\mathrm{GF}(2)$ has the connection polynomial $\prod_{i=1}^{4} p_{i}(x)$ with order $\theta=\operatorname{lcm}\left(\theta_{1}, \theta_{2}, \theta_{3}, \theta_{4}\right) \approx 2^{125}$ (by Lemma $6.50,[18$, p.214]) and degree $L=\sum_{i=1}^{4} L_{i}=128$.

\subsection{Finding the Multiple Polynomial with Low Weight}

Let $d_{0}$ be the degree of a general polynomial $p(x)$. We use the standard approximation to estimate the minimal weight $w_{d}$ of multiples of $p(x)$ with degree at most $d$ by the following constraint: $w_{d}$ is the smallest $w$ such that

$$
\frac{1}{2^{d_{0}}} \times\left(\begin{array}{c}
d \\
w-1
\end{array}\right) \geq 1 .
$$

Listed in Table 3 is the estimated ${ }^{3} w_{d}$ corresponding to $d$ with $p(x)=\prod_{i=1}^{4} p_{i}(x)$ $\left(d_{0}=128\right)$ by solving Inequality $(5)$.

To find multiples with low weight, efficient algorithms like [4] exist provided the degree is low, say, less than 2000, which does not apply to E0. So we can use the conventional birthday paradox to find $Q(x)$ with the minimal $d$ (i.e. $w=w_{d}$ ), which takes precomputation time $P T \approx O\left(d^{\left\lceil\frac{w-1}{2}\right\rceil}\right)$; or we apply the generalized birthday problem [29] to find $Q(x)$ of same weight but higher degree with much less precomputation as tradeoff. Table 4 compares the two algorithms. In Appendix $\mathrm{B}$, we also provide some non-optimal multiples as examples, including $Q_{4}(x)$ with $w=4$ and $d \approx 2^{65}$.

Table 3. The estimated minimal weight $w_{d}$ of multiples of $p_{1}(x) p_{2}(x) p_{3}(x) p_{4}(x)$ with degree $d$ by (5)

\begin{tabular}{|c|c|c|c|c|c|c|c|c|c|c|c|c|c|}
\hline$d$ & 128 & 247 & 458 & 855 & 1749 & 2387 & $2^{18}$ & $2^{23}$ & $2^{27}$ & $2^{33}$ & $2^{44}$ & $2^{65}$ & $\theta$ \\
\hline$w_{d}$ & $=49$ & $\approx 31$ & $\approx 24$ & $\approx 20$ & $\approx 17$ & $\approx 16$ & $\approx 9$ & $\approx 7$ & $\approx 6$ & $\approx 5$ & $\approx 4$ & $\approx 3$ & $=2$ \\
\hline
\end{tabular}

\subsection{Building a Uni-Bias-Based Distinguisher for E0}

Let $Q(x)=\sum_{i=1}^{w} x^{q_{i}}$ be the normalized multiple of $\prod_{i=1}^{4} p_{i}(x)$ with degree $d$ and weight $w$, where $0=q_{1}<q_{2}<\ldots<q_{w}=d$. As $\oplus_{i=1}^{w} y_{t_{0}+q_{i}}^{1}=0$ holds for all $t_{0}$, by Eq.(1), we deduce

$$
\oplus_{i=1}^{w}\left(z_{t_{0}+q_{i}+5} \oplus z_{t_{0}+q_{i}}\right)=\oplus_{i=1}^{w}\left(c_{t_{0}+q_{i}+5}^{0} \oplus c_{t_{0}+q_{i}}^{0}\right) .
$$

\footnotetext{
${ }^{3}$ Two special cases occur for $d=d_{0}$ and $d=\theta$ because we know the exact value of $w_{d}$.
} 
Table 4. Complexity of finding multiple of $p_{1}(x) p_{2}(x) p_{3}(x) p_{4}(x)$ with degree $d$, weight $w$

\begin{tabular}{||c||c|c|c|c|c|c|c||c||||}
\cline { 2 - 9 } \multicolumn{1}{c||}{} & \multicolumn{6}{c||}{ birthday problem } \\
\cline { 2 - 8 } \multicolumn{1}{c||}{ with minimal $d$} & \multicolumn{1}{c|}{ tradeoff } \\
\hline \hline$d$ & $2^{18}$ & $2^{23}$ & $2^{27}$ & $2^{33}$ & $2^{44}$ & $2^{65}$ & $2^{32}$ & $2^{43}$ \\
\hline$w$ & 9 & 7 & 6 & 5 & 4 & 3 & 9 & 5 \\
\hline $\log _{2} P T$ & 72 & 69 & 68 & 66 & 66 & 65 & 35 & 45 \\
\hline
\end{tabular}

By the Piling-up Lemma [20] and Corollary 7, we know the right-hand side of Eq.(6) is equal to zero with probability $\frac{1}{2}+\frac{1}{2} \cdot \gamma^{w}$. With standard linear cryptanalysis techniques, we can distinguish the keystream $\left\{z_{t}\right\}$ of E0 from a truly random sequence with $\gamma^{-2 \cdot w}$ samples, simply by checking the left-hand side of Eq.(6) equals zero most of the time. Based on $Q(x)$ with $d$ and $w$, we minimize the data complexity $n$ by choosing $n=\gamma^{-2 \cdot w}+d$. Table 5 shows the minimum $n=2^{34}$ is achieved with $d=2^{33}, w=5$. Table 6 summarizes the best performance of our primary distinguisher for E0 based on either the use of $Q_{4}(x)$ with weight 4 in Appendix B, or a search of $Q(x)$.

Table 5. Data complexity of the primary distinguisher for E0

\begin{tabular}{|c||c|c|c|c|c|c|c|c|c|c|c|c||c|c|}
\hline$d$ & $L$ & 247 & 458 & 855 & 1749 & 2387 & $2^{18}$ & $2^{23}$ & $2^{27}$ & $2^{33}$ & $2^{44}$ & $2^{65}$ & $2^{32}$ & $2^{43}$ \\
\hline$w$ & 49 & 31 & 24 & 20 & 17 & 16 & 9 & 7 & 6 & 5 & 4 & 3 & 9 & 5 \\
\hline $\log _{2} n$ & 329 & 209 & 162 & 135 & 115 & 108 & 61 & 47 & 41 & 34 & 44 & 65 & 61 & 43 \\
\hline
\end{tabular}

Table 6. Summary of the best primary distinguisher for E0

\begin{tabular}{||c||c|c|c|c|c||}
\hline \multicolumn{1}{|c||}{ Type } & $d$ & $w$ & Precomputation & Data & Time \\
\hline \hline use $Q(x)=Q_{4}(x)$ & $2^{65}$ & 4 & - & $2^{65}$ \\
\hline \hline find $Q(x)$ & minimal $d$ & $2^{33}$ & 5 & $2^{66}$ & $2^{34}$ \\
\cline { 2 - 6 } with & tradeoff & $2^{43}$ & 5 & $2^{45}$ & $2^{43}$ \\
\hline
\end{tabular}

\section{The Advanced Multi-bias-Based Distinguisher for E0}

\subsection{Preliminaries}

Definition 8. Given $f, g: G F(2)^{\ell} \rightarrow \mathbf{R}$, for $a \in G F(2)^{\ell}$, we define

1. $(f \otimes g)(a)=\sum_{b \in G F(2)^{\ell}} f(b) \cdot g(a \oplus b) ; f^{\otimes w}(a)=(\underbrace{f \otimes \cdots \otimes f}_{w \text { times }})(a)$

2. $\hat{f}(a)=\sum_{b \in G F(2)^{\ell}}(-1)^{a \cdot b} f(b)$

3. $\|f\|=\sqrt{\sum_{a \in G F(2)^{\ell}} f^{2}(a)}$

4. $\Delta(f)=2^{\frac{\ell}{2}}\left\|f-\frac{1}{2^{\ell}} \cdot \mathbf{1}\right\|$, where $\mathbf{1}$ denotes a constant function equal to 1 
Note that the first two definitions correspond to convolution and Walsh transform respectively. We recall these basic facts: for any $f, g: G F(2)^{\ell} \rightarrow \mathbf{R}$, we have

$-\widehat{f \otimes g}(a)=\hat{f}(a) \cdot \hat{g}(a)$, for $a \in G F(2)^{\ell}$;

$-2^{\ell}\|f\|^{2}=\|\hat{f}\|^{2}$

- if $f$ is a distribution, i.e. $\sum_{a} f(a)=1$ and $f(a) \geq 0$ for all $a \in G F(2)^{\ell}$, then the distribution of the XOR of $w$ i.i.d. random vectors with distribution $f$ is $f^{\otimes w}$, moreover, $\Delta^{2}(f)=\sum_{a \neq \mathbf{0}} \hat{f}^{2}(a)$;

- If the random Boolean variable $A$ follows the distribution $f$, then $\Delta(f)=$ $\Delta(A)$, where $\Delta(A)$ is defined in Definition 4 .

\subsection{An Efficient Way to Deploy Multi-biases in E0 Simultaneously}

Given a linear mapping $h: G F(2)^{\ell} \rightarrow G F(2)^{r}$ of rank $r$, we define $r$-bit vectors $A_{t}=h\left(c_{r t}^{0}, \ldots, c_{r t+\ell-1}^{0}\right)$ and $B_{t}=\oplus_{i=1}^{w} A_{t+q_{i}}$. Note that $B_{t}$ can be derived from the keystream $\left\{z_{t}\right\}$ directly. Except for accidentally bad choices of $h$, we make a heuristic assumption that all $A_{t}$ 's are independent. Let $\mathcal{D}$ be the probability distribution of the $\ell$-bit vector $\left(c_{r t}^{0}, \ldots, c_{r t+\ell-1}^{0}\right)$, and let $\mathcal{D}_{A}$ be the probability distribution of the $r$-bit vector $A_{t}$. The Walsh transforms of $\mathcal{D}_{A}$ and $\mathcal{D}$ are linked by

$$
\hat{\mathcal{D}}_{A}(b)=\hat{\mathcal{D}}\left(h^{t}(b)\right) \text {, for all } \mathrm{b} \in G F(2)^{r} .
$$

Now we discuss how to design $h$ in order to reduce data complexity. From Baignères [2, Theorem 3, p.10], we know that we can distinguish a distribution $f$ of $r$-bit random vectors from a uniform distribution with $1 / \Delta^{2}(f)$ samples. Here, the distribution of $B_{t}$ is $f=\mathcal{D}_{A}^{\otimes w}$. So the modified distinguisher needs data complexity

$$
n=\frac{r}{\Delta^{2}\left(\mathcal{D}_{A}^{\otimes w}\right)}+d \text { (bits). }
$$

Let $k$ be the number of the largest Walsh coefficients $\hat{\mathcal{D}}_{A}(b)$ over all nonzero $b$ with absolute value ${ }^{4} \eta$. Since $\Delta^{2}\left(\mathcal{D}_{A}^{\otimes w}\right) \approx k \eta^{2 w}$, we obtain

$$
n \approx \frac{r}{k} \eta^{-2 w}+d
$$

In order to lower $n$, it's necessary to have $r<k$. This implies the $k$ largest coefficients are linearly dependent, which happens to be true in E0: recall that the 6 -bit vectors of the three largest biases satisfy the linear relation,

$$
(1,1,1,1,1,0) \oplus(0,1,1,1,1,1)=(1,0,0,0,0,1) .
$$

As a simple solution we may just pick $\ell=6, r=2, \alpha_{1}=(1,1,1,1,1,0)$ and $\alpha_{2}=(0,1,1,1,1,1)$ (where $\alpha_{i}$ denotes the $i$-th row of $h$ ), then we obtain

\footnotetext{
${ }^{4}$ Note that from Subsection 4.3 we have $\eta \leq \gamma$ for $\ell \leq 26$ regardless of $r$ and $h$.
} 
$k=3$. And $n$ is reduced to a factor of $\frac{2}{3}$ for negligible $d$. Indeed, recall that we proved by computation that the largest Walsh coefficient for $\ell \leq 26$ are either $(0, \ldots, 0,1,1,1,1,1,0, \ldots, 0)$ or $(0, \ldots, 0,1,0,0,0,0,1,0, \ldots, 0)$. Thus $k \leq(\ell-4)+(\ell-5)=$ $2 \ell-9$. This leads to a more general solution, if we pick $\ell=r+4$, and the $i$-th row of $h$ as

$$
\alpha_{i}=(\underbrace{0, \ldots, 0}_{i-1 \text { zeros }}, 1,1,1,1,1, \underbrace{0, \ldots, 0}_{\ell-i-4 \text { zeros }}) \text { for } i=1, \ldots, r,
$$

then we obtain $k=2 r-1$. And so the improved factor $\frac{r}{2 r-1}$ of data complexity tends to $\frac{1}{2}$ for negligible $d$ when $r$ goes to infinity; however, because of the underlying assumption for E0, $\ell$ is restricted to no larger than 26, i.e. $r \leq 22$. To conclude, we show that the modified distinguisher (Algorithm 1) needs data complexity

$$
n \approx \frac{r}{2 r-1} \cdot \gamma^{-2 w}+d, \text { for } 1 \leq r \leq 22 .
$$

Observe that Section 4 actually deals with the special case of $r=1$. Table 7 shows the best improvement achieved with $r=22$. We see that the minimum $n$ drops from previous $2^{34}$ to $2^{33}$.

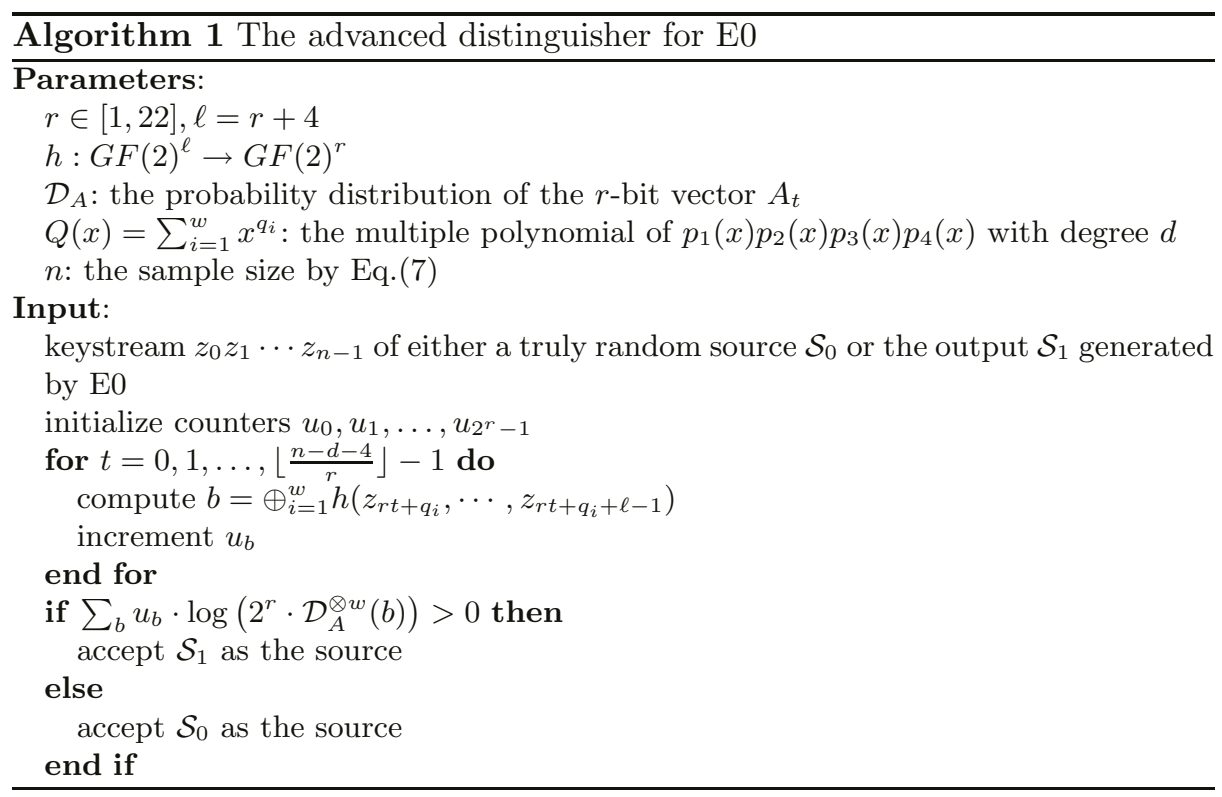

\section{A Maximum Likelihood Decoding Algorithm}

We restate the MLD problem for a general linear code (see [19] for details) of length $n$ and dimension $L$ with generator matrix $G$ (let $G_{t}$ denote the $t$-th 
Table 7. Data complexity of the advanced distinguisher for E0

\begin{tabular}{|c||c|c|c|c|c|c|c|c|c|c|c|c||c|c|}
\hline$d$ & $L$ & 247 & 458 & 855 & 1749 & 2387 & $2^{18}$ & $2^{23}$ & $2^{27}$ & $2^{33}$ & $2^{44}$ & $2^{65}$ & $2^{32}$ & $2^{43}$ \\
\hline$w$ & 49 & 31 & 24 & 20 & 17 & 16 & 9 & 7 & 6 & 5 & 4 & 3 & 9 & 5 \\
\hline $\log _{2} n$ & 328 & 208 & 161 & 134 & 114 & 107 & 60 & 46 & 40 & 33 & 44 & 65 & 60 & 43 \\
\hline
\end{tabular}

column vector of $G)$ : find the closest codeword $\left(x_{1}, \ldots, x_{n}\right)$ to the received vector $\left(s_{1}, \ldots, s_{n}\right)$, and decode the message $\mathbf{r}=\left(r_{1}, \ldots, r_{L}\right)$ such that $x_{t}=\mathbf{r} G_{t}$, i.e. find such $\mathbf{r}$ that minimizes $N(\mathbf{r})=\sum_{t=1}^{n}\left(s_{t} \oplus x_{t}\right)$.

\subsection{The Time-Domain Analysis}

Obviously, the trivial approach (yet common in most correlation attacks) to find $\mathbf{r}$ is an exhaustive search in time-domain: for every message $\tilde{\mathbf{r}}$, we compute the distance $N(\tilde{\mathbf{r}})$ and keep the smallest. The final record leads to $\mathbf{r}$. The time complexity is $O\left(n \cdot 2^{L}\right)$ with memory $n$-bits.

\subsection{The Frequency-Domain Analysis}

We introduce an integer-valued function,

$$
\mathcal{W}(x)=\sum_{1 \leq t \leq n: G_{t}=x^{\top}}(-1)^{s_{t}},
$$

for all $x \in G F(2)^{L}$, where $\top$ denotes the matrix transpose. We compute the Walsh transform $\hat{\mathcal{W}}$ of $\mathcal{W}$ as follows:

$$
\hat{\mathcal{W}}(\mathbf{r})=\sum_{x \in G F(2)^{L}}(-1)^{\mathbf{r} \cdot x} \mathcal{W}(x)=\sum_{t=1}^{n}(-1)^{s_{t} \oplus \mathbf{r} G_{t}}=\sum_{t=1}^{n}(-1)^{s_{t} \oplus x_{t}}=n-2 N(\mathbf{r}) .
$$

We thereby reach the theorem below.

\section{Theorem 9.}

$$
N(\mathbf{r})=\frac{1}{2}(n-\hat{\mathcal{W}}(\mathbf{r})),
$$

for all $\mathbf{r} \in G F(2)^{L}$, where $\mathcal{W}$ is defined by Eq.(8).

This generalizes the result $[19, \mathrm{p} .414]$ of a special case when $n=2^{L}$ and $G_{t}^{\top}$ corresponds to the binary representation of $t$. So we just compute the table of $\mathcal{W}$, perform FWT [30], and find the maximal $\hat{\mathcal{W}}(\mathbf{r})$. The time and memory complexities of FWT are $O\left(L \cdot 2^{L}\right), O\left(2^{L}\right)$ respectively. Since the precomputation of $\mathcal{W}$ takes time $O(n)$ with memory $O(n)$, we conclude that our improved MLD algorithm runs in $O\left(n+L \cdot 2^{L}\right)$ with memory $O\left(2^{L}\right)$ (additionally, using linear transformation allows to compute FWT over $G F(2)^{k}$ with memory $O\left(2^{k}\right)$ where $\left.k=\left\lceil\log _{2} n\right\rceil\right)$. Note that when $n \geq 2^{L}$, the time complexity corresponds to $O(n)$, which is optimal in the sense that it stands on the same order of magnitude 
as the data complexity does. Table 8 compares the original exhaustive search algorithm with the improved frequency transformation algorithm. Note that the technique of FWT was used in another context [7] to speed up other kinds of fast correlation attacks. In the next section we will see how it helps to speed up the attack [10] by a factor of $2^{24}$. We estimate similar correlation attacks like [6] can be speeded up by a factor of 10; undoubtedly, some other attacks can be significantly improved by our algorithm as well.

Table 8. Maximum likelihood decoding algorithms

\begin{tabular}{||c||c|c||}
\cline { 2 - 3 } \multicolumn{1}{c|}{} & time & memory \\
\hline \hline Exhaustive Search & $n \cdot 2^{L}$ & $n$ \\
\hline Frequency Transformation & $n+L \cdot 2^{L}$ & $\min \left(n, 2^{L}\right)$ \\
\hline
\end{tabular}

\subsection{A More Generalized MLD Algorithm}

We further generalize the preceding problem by finding the $L$-bit vector $\mathbf{r}$ such that given a sequence of $\ell$-bit vectors $S_{1}, \ldots, S_{k}$ and $f: G F(2)^{\ell} \rightarrow \mathbf{R}$ together with matrices $G_{1}, \ldots, G_{k}$ of size $L$ by $\ell$, the sequence of $\ell$-bit vectors $X_{1}, \ldots, X_{k}$ defined by $X_{t}=\mathbf{r} G_{t}$ minimizes $N(\mathbf{r})=\sum_{t=1}^{k} f\left(S_{t} \oplus X_{t}\right)$. Note that previous subsections are merely a special case of $\ell=1, k=n$ and $f(a)=a$ for $a \in G F(2)$.

Define a real function $\mathcal{W}$ by:

$$
\mathcal{W}(x)=\frac{1}{2^{\ell}} \sum_{1 \leq t \leq k, a \in G F(2)^{\ell}: a G_{t}^{\top}=x}(-1)^{a \cdot S_{t}} \hat{f}(a),
$$

for all $x \in G F(2)^{L}$. We compute the Walsh transform $\hat{\mathcal{W}}$ of $\mathcal{W}$ as follows:

$$
\begin{aligned}
\hat{\mathcal{W}}(\mathbf{r}) & =\sum_{x \in G F(2)^{L}}(-1)^{\mathbf{r} \cdot x} \mathcal{W}(x) \\
& =\frac{1}{2^{\ell}} \sum_{t=1}^{k} \sum_{a \in G F(2)^{\ell}}(-1)^{a \cdot\left(\mathbf{r} G_{t} \oplus S_{t}\right)} \hat{f}(a) \\
& =\sum_{t=1}^{k} f\left(\mathbf{r} G_{t} \oplus S_{t}\right) \\
& =N(\mathbf{r}) .
\end{aligned}
$$

Algorithm 2 directly follows above computation. The total running time of our algorithm is $O\left(k \ell L 2^{\ell}+L 2^{L}\right)$ with memory $O\left(2^{L}\right)$. To speed up the computation of $\mathcal{W}$, we could precompute the inner products of all pairs of $\ell$-bit vectors in time $O\left(2^{2 \ell}\right)$ with memory $O\left(2^{2 \ell}\right)$. Thus, the total running time of the algorithm is $O\left(2^{2 \ell}+k L 2^{\ell}+L 2^{L}\right)$ with memory $O\left(2^{2 \ell}+2^{L}\right)$. 


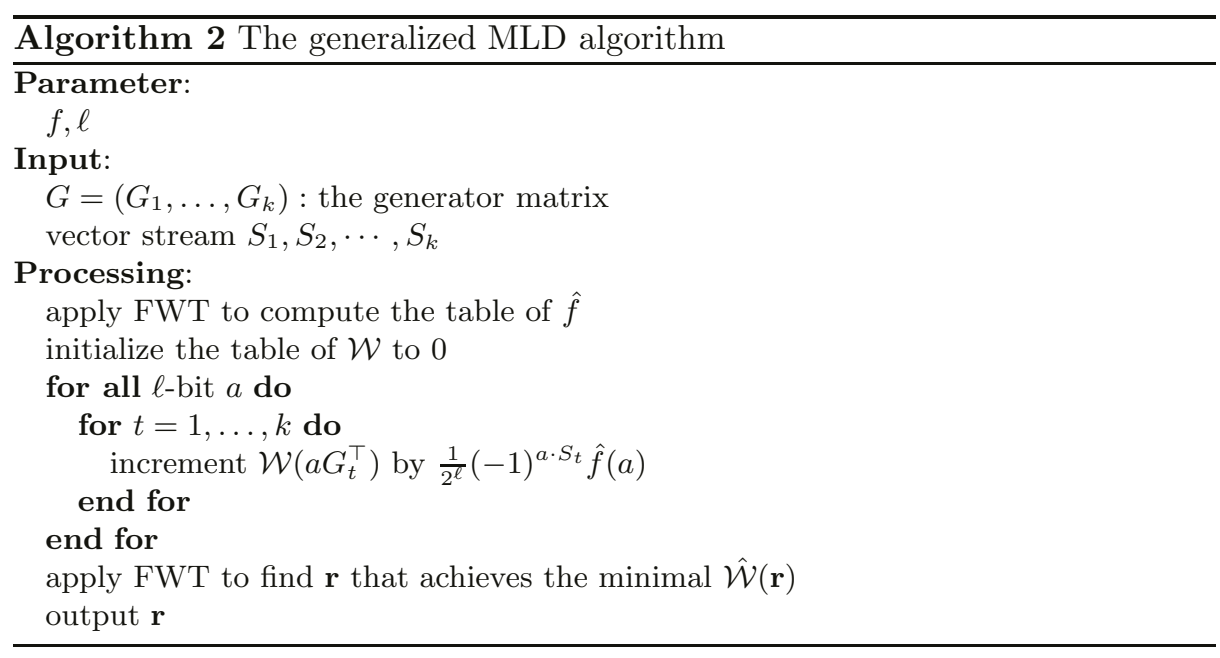

In the special case that is applicable to E0 (as is done in the next section): $G_{t+1}=A G_{t}$ for $t=1, \ldots, k$, we precompute another table to map any $L$ bit vector $x$ to $x A^{\top}$. It takes time $2^{L}$ with memory $2^{L}$. The total time of the algorithm is thus $O\left(2^{2 \ell}+(L+k) 2^{\ell}+L 2^{L}\right)$, with memory $O\left(2^{2 \ell}+2^{L}\right)$.

\section{The Key-Recovery Attack Against E0}

We approach similarly as in [10] to transform our distinguisher of Subsection 4.3 into a key-recovery attack. Our main contribution, however, is to decrease the time complexity by applying the preceding algorithm.

Let $Q(x)=\sum_{i=1}^{w} x^{q_{i}}$ be the multiple polynomial of $p_{2}(x) p_{3}(x) p_{4}(x)$ with degree $d$ and weight $w$. Using techniques in Subsection 4.2 to find $Q(x)$ with (precomputation) complexity $P C$, we list the corresponding triplets $(w, d, P C)$ for small $w$ in Table 9 .

Table 9. Complexity of finding multiple of $p_{2}(x) p_{3}(x) p_{4}(x)$ with degree $d$, weight $w$

\begin{tabular}{||c||c|c|c|c||c||}
\cline { 2 - 5 } \multicolumn{1}{c|}{} & \multicolumn{4}{c||}{ birthday problem } \\
\cline { 2 - 6 } \multicolumn{1}{c|}{} & \multicolumn{3}{c||}{ with min. $d$} & tradeoff \\
\hline \hline weight $w$ & 5 & 4 & 3 & 2 & 5 \\
\hline degree $d$ & $2^{27}$ & $2^{36}$ & $2^{52}$ & $2^{100}$ & $2^{34.3}$ \\
\hline Precomputation $P C$ & $2^{54}$ & $2^{54}$ & $2^{52}$ & - & $2^{36.3}$ \\
\hline
\end{tabular}

Let $\tilde{\mathbf{x}}^{\mathbf{1}}$ be a guess for $\mathbf{x}^{\mathbf{1}}$, the initial state of $R_{1}$ which generates the keystream $\left\{z_{t}\right\}$ together with the other three fixed LFSRs. Denote $\tilde{x}_{t}^{1}$ the output bit of $R_{1}$ with the initial state $\tilde{\mathbf{x}}^{\mathbf{1}}$ at time $t$. We define

$$
b_{t}\left(\tilde{\mathbf{x}}^{\mathbf{1}}\right)=\oplus_{i=1}^{w}\left(z_{t+q_{i}} \oplus z_{t+q_{i}+5}\right) \oplus \oplus_{i=1}^{w}\left(\tilde{x}_{t+q_{i}}^{1} \oplus \tilde{x}_{t+q_{i}+5}^{1}\right) .
$$


It can be shown that the second addend in Eq.(9) is also an $m$-sequence generated by the same LFSR. For brevity, we set

$$
\begin{aligned}
& r_{t}=\oplus_{i=1}^{w}\left(\tilde{x}_{t+q_{i}}^{1} \oplus \tilde{x}_{t+q_{i}+5}^{1}\right), \\
& s_{t}=\oplus_{i=1}^{w}\left(z_{t+q_{i}} \oplus z_{t+q_{i}+5}\right),
\end{aligned}
$$

for $t=1, \ldots, n$ (it corresponds to the data complexity $n+d$ ). We rewrite Eq.(9) as

$$
b_{t}\left(\tilde{\mathbf{x}}^{\mathbf{1}}\right)=s_{t} \oplus r_{t} .
$$

Given $n$-bit sequence of $b_{t}\left(\tilde{\mathbf{x}}^{\mathbf{1}}\right)^{\prime}$ 's, we count the occurrences ${ }^{5} N\left(\tilde{\mathbf{x}}^{\mathbf{1}}\right)$ of ones, i.e. $N\left(\tilde{\mathbf{x}}^{\mathbf{1}}\right)=\sum_{t=0}^{n-1} b_{t}\left(\tilde{\mathbf{x}}^{\mathbf{1}}\right)$. Using the analysis of [28], we estimate $N\left(\mathbf{x}^{\mathbf{1}}\right)$ is the smallest of all $N\left(\tilde{\mathbf{x}}^{\mathbf{1}}\right)$ with

$$
n \approx \frac{4 L_{1} \log 2}{\gamma^{2 w}}
$$

Note that this estimated figure is actually comparable to the conventional estimation $[6,16]$ on critical data complexity $n_{0}$ in correlation attacks, where

$$
n_{0}=\frac{L_{1}}{1-h\left(\frac{1}{2}+\frac{1}{2} \gamma^{w}\right)} \approx \frac{2 L_{1} \log 2}{\gamma^{2 w}}
$$

and $h$ is the binary entropy function ${ }^{6}$. According to [6] simulations showed the probability of success is close to 1 (resp. $\frac{1}{2}$ ) for $n=2 n_{0}$ (resp. $n=n_{0}$ ) which is consistent with our analysis. Table 10 shows our estimated minimal $n$ for $N\left(\mathbf{x}^{\mathbf{1}}\right)$ to achieve a top rank corresponding to $w$. Now define $G_{t}=\left(a_{0}, \ldots, a_{L_{1}-1}\right)^{\top}$, where $a_{0}+a_{1} x+\cdots+a_{L_{1}-1} x^{L_{1}-1}=x^{t} \bmod p_{1}(x)$. Clearly our current problem to recover $R_{1}$ right fits into the MLD problem in Subsection 6.2. So we use the preceding MLD algorithm to recover $\mathbf{r}$ first, then apply linear transform to solve $\mathbf{x}^{\mathbf{1}}$. Finally we conduct the same analysis as in Section 5 to decrease data complexity down to $O\left(\frac{r}{2 r-1} \times \frac{4 L_{1} \log 2}{\gamma^{2 w}}\right)$; and we apply the technique introduced in Subsection 6.3 to obtain the reduced time complexity $O\left(n+\theta_{1} \cdot 2^{r}+L_{1} \cdot 2^{L_{1}}\right)$. So, choosing $r=12$, we can halve the time and data complexities. The attack complexities to recover $R_{1}$ for E0 are listed in Table 11 . Once we recover $R_{1}$, we target $R_{2}$ next based on multiple of $p_{3}(x) p_{4}(x)$. Last, we use the technique of guess and determine in [11] to solve $R_{3}$ and $R_{4}$ with knowledge of the shortest two LFSRs. The detailed complexities of each step are shown in Table 12. A comparison of our attacks with the similar attack ${ }^{7}[10]$ and the best two algebraic attacks $[1,8]$ is shown in Table 13 .

\footnotetext{
${ }^{5} w$ is fixed in the attack, so we omit it in the notation $N\left(\tilde{\mathbf{x}}^{\mathbf{1}}\right)$.

${ }^{6} h(p)=-p \log _{2} p-(1-p) \log _{2}(1-p)$ for $0<p<1$.

7 The estimate of data complexity in [10] uses a different heuristic formula than ours. However we believe that their estimate and ours in Attack B are essentially the same.
} 
Table 10. The estimated minimal $n$ for $N\left(\mathbf{x}^{\mathbf{1}}\right)$ to top the rank from Eq.(10)

\begin{tabular}{|c|c|c|c|c|c|}
\hline weight $w$ & 5 & 4 & 3 & 2 & 1 \\
\hline$n$ & $2^{40}$ & $2^{33}$ & $2^{27}$ & $2^{20}$ & $2^{14}$ \\
\hline
\end{tabular}

Table 11. Summary of primary partial key-recovery attacks against $R_{1}$ for E0

\begin{tabular}{||l||c|c|c|c|c|c|c||}
\cline { 2 - 7 } \multicolumn{1}{c|}{} & $w$ & $d$ & $n$ & data & precomputation & time & memory \\
\hline \hline Attack A & 5 & $2^{34.3}$ & $2^{39}$ & $2^{39}$ & $2^{36.3}$ & $2^{39}$ & $2^{25}$ \\
\hline Attack B & 4 & $2^{36}$ & $2^{33}$ & $2^{36}$ & $2^{54}$ & $2^{36}$ & $2^{25}$ \\
\hline
\end{tabular}

Table 12. Detailed complexities of our key-recovery attack against E0

\begin{tabular}{||c||c|c|c|c|c|c|c||}
\cline { 2 - 7 } \multicolumn{1}{c||}{} & $w$ & $d$ & $n$ & data & precomputation & time & memory \\
\hline \hline$R_{1}$ & 5 & $2^{34.3}$ & $2^{39}$ & $2^{39}$ & $2^{36.3}$ & $2^{39}$ & $2^{25}$ \\
\hline$R_{2}$ & 3 & $2^{36}$ & $2^{27}$ & $2^{36}$ & $2^{37}$ & $2^{36}$ & $2^{27}$ \\
\hline$R_{3}$ and $R_{4}$ & - & - & - & 76 & - & $2^{33}$ & - \\
\hline \hline total & - & - & - & $2^{39}$ & $2^{37}$ & $2^{39}$ & $2^{27}$ \\
\hline
\end{tabular}

Table 13. Complexities comparison of our attacks with the similar attack and algebraic attacks

\begin{tabular}{|c|c|c|c|c|c|}
\hline & & Precomputation & Time & Data & Memory \\
\hline Algebraic & & 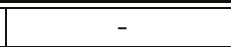 & $2^{67.58}$ & $2^{23.07}$ & $2^{46.14}$ \\
\hline Attacks & [8] & $2^{28}$ & $2^{49}$ & $2^{23.4}$ & $2^{37}$ \\
\hline Attack [1 & & $2^{54}$ & $2^{63}$ & $2^{34}$ & $2^{34}$ \\
\hline Our & $\overline{\mathrm{A}}$ & $2^{37}$ & $2^{39}$ & $2^{39}$ & $2^{27}$ \\
\hline Attacks & $\mathrm{B}$ & $2^{54}$ & $2^{37}$ & $2^{36}$ & $2^{27}$ \\
\hline
\end{tabular}

\section{Conclusions}

This paper formulates a systematic computation method of correlations by a recursive expression, which makes it easier to calculate correlations of the FSM output sequences up to 26 bits for E0 (and allows us to prove for the first time that the two known biases are the only largest). Then we successfully apply the concept of convolution to the analysis of the distinguisher based on all correlations, which allows us to build an efficient distinguisher that halves the data complexity of the basic uni-bias-based distinguisher due to the linear dependency of the two largest biases. Finally, by means of FWT, we propose a novel MLD algorithm to recover the closest codeword for any linear code. Our proposed algorithm can be easily adapted to speed up a class of fast correlation attacks. Furthermore the algorithm is optimal when the length $n$ of the code and the dimension $L$ satisfy the relation $n \geq 2^{L}$, which is the case when we apply it to recover $R_{1}$ for $\mathrm{E} 0$. This results in the best known key-recovery attack against E0. Considering a maximal keystream length of 2745 bits for practical E0 in 
Bluetooth, our results still remain the academic interest. Meanwhile, our attack successfully illustrates the attack methodology of Baignères et al. ${ }^{8}$

\section{Acknowledgment}

Words fail the first author when she would like to express her heartfelt thanks to her forever faithful bosom friend Aoife Hegarty, who, in the face of a rough time in her own life, has been generously offering continuous one-way help through all those years...

\section{References}

1. F. Armknecht, M. Krause, Algebraic Attacks on Combiners with Memory, Advances on Cryptography - CRYPTO 2003, Lecture Notes in Computer Science, vol.2729, D. Boneh ed., Springer-Verlag, pp. 162-175, 2003

2. T. Baignères, A Generalization of Linear Cryptanalysis, Diploma Thesis, EPFL, 2003

3. Bluetooth ${ }^{\mathrm{TM}}$, Bluetooth Specification, version 1.2, pp. 903-948, November, 2003, available at http://www.bluetooth.org

4. A. Canteaut, F. Chabaud, A New Algorithm for Finding Minimum-weight Words in a Linear Code: Application to Primitive Narrow-sense BCH Codes of Length 511, INRIA, technical report, No. 2685, 1995

5. A. Canteaut, M. Trabbia, Improved Fast Correlation Attacks Using Parity-check Equations of Weight 4 and 5, Advances in Cryptology - EUROCRYPT 2000, Lecture Notes in Computer Science, vol.1807, B. Preneel ed., Springer-Verlag, pp. $573-588,2000$

6. V. Chepyzhov, T. Johansson, B. Smeets, A Simple Algorithm for Fast Correlation Attacks on Stream Ciphers, Fast Software Encryption 2000, Lecture Notes in Computer Science, vol.1978, B. Schneier ed., Springer-Verlag, pp. 181-195, 2000

7. P. Chose, A. Joux, M. Mitton, Fast Correlation Attacks: An Algorithmic Point of View, Advances in Cryptology - EUROCRYPT 2002, Lecture Notes in Computer Science, vol.2332, L. R. Knudsen ed., Springer-Verlag, pp. 209-221, 2002

8. N. T. Courtois, Fast Algebraic Attacks on Stream Ciphers with Linear Feedback, Advances on Cryptography - CRYPTO 2003, Lecture Notes in Computer Science, vol.2729, D. Boneh ed., Springer-Verlag, pp. 176-194, 2003

9. P. Ekdahl, T. Johansson, Some Results on Correlations in the Bluetooth Stream Cipher, Proceedings of the 10th Joint Conference on Communications and Coding, Austria, 2000

10. P. Ekdahl, On LFSR Based Stream Ciphers (Analysis and Design), Ph.D. Thesis, Lund Univ., Nov. 2003

11. S. Fluhrer, S. Lucks, Analysis of the E0 Encryption System, Selected Areas in Cryptography- SAC 2001, Lecture Notes in Computer Science, vol. 2259, S. Vaudenay and A. Youssef eds., Springer-Verlag, pp. 38-38, 2001

12. J. D. Golić, Correlation Properties of a General Binary Combiner with Memory, Journal of Cryptology, vol. 9, pp. 111-126, Nov. 1996

\footnotetext{
${ }^{8}$ Personal communication. Prior work available in [2].
} 
13. J. D. Golić, V. Bagini, G. Morgari, Linear Cryptanalysis of Bluetooth Stream Cipher, Advances in Cryptology - EUROCRYPT 2002, Lecture Notes in Computer Science, vol. 2332, L. R. Knudsen ed., Springer-Verlag, pp. 238-255, 2002

14. M. Hermelin, K. Nyberg, Correlation Properties of the Bluetooth Combiner, Information Security and Cryptology- ICISC'99, Lecture Notes in Computer Science, vol. 1787, JooSeok. Song ed., Springer-Verlag, pp. 17-29, 2000

15. M. Jakobsson, S. Wetzel, Security Weakness in Bluetooth, Topics in Cryptology - CT-RSA 2001, Lecture Notes in Computer Science, vol. 2020, D. Naccache ed., Springer-Verlag, pp. 176-191, 2001

16. T. Johansson, F. Jonsson, Improved Fast Correlation Attacks on Stream Ciphers via Convolutional Codes, Advances on Cryptography - CRYPTO'99, Lecture Notes in Computer Science, vol.1666, M. Wiener ed., Springer-Verlag, pp. 181-197, 1999

17. M. Krause, BDD-Based Cryptanalysis of Keystream Generators, Advances in Cryptology - EUROCRYPT 2002, Lecture Notes in Computer Science, vol. 2332, L. R. Knudsen ed., Springer-Verlag, pp. 222-237, 2002

18. R. Lidl, H. Niederreiter, Introduction to Finite Fields and Their Applications, Cambridge, 1986

19. F. J. MacWilliams, N. J. A. Sloane, The Theory of Error-correcting Codes, NorthHolland, 1996

20. M. Matsui, Linear Cryptanalysis Method for DES Cipher, Advances in Cryptology - EUROCRYPT'93, Lecture Notes in Computer Science, vol.765, Springer-Verlag, pp. 386-397, 1993

21. W. Meier, O. Staffelbach, Fast Correlation Attacks on Certain Stream Ciphers, Journal of Cryptology, vol. 1, pp. 159-176, Nov. 1989

22. W. Meier, O. Staffelbach, Correlation Properties of Combiners with Memory in Stream Ciphers, Journal of Cryptology, vol. 5, pp. 67-86, Nov. 1992

23. A. J. Menezes, P. C. van. Oorschot, S. A. Vanstone, Handbook of Applied Cryptography, CRC, 1996

24. W. Penzhorn, Correlation Attacks on Stream Ciphers: Computing Low Weight Parity Checks based on Error Correcting Codes, Fast Software Encryption 96, Lecture Notes in Computer Science, vol.1039, D. Gollmann ed., Springer-Verlag, pp. 159172, 1996

25. R. A. Rueppel, Correlation Immunity and the Summation Generator, Advances in Cryptology - CRYPTO'85, Lecture Notes in Computer Science, Springer-Verlag, pp. 260-272, 1986

26. M. Saarinen, Re: Bluetooth and E0, Posted at sci.crypt.research, 02/09/00

27. T. Siegenthaler, Correlation-Immunity of Nonlinear Combining Functions for Cryptographic Applications, IEEE Transactions on Information Theory, vol. 30, pp. $776-780,1984$

28. S. Vaudenay, An Experiment on DES - Statistical Cryptanalysis, Proceedings of the 3rd ACM Conferences on Computer Security, pp. 139-147, 1996

29. D. Wagner, A Generalized Birthday Problem, Advances in Cryptology - CRYPTO 2002, Lecture Notes in Computer Science, vol.2442, Springer-Verlag, pp. 288-304, 2002

30. R. K. Yarlagadda, J. E. Hershey, Hadamard Matrix Analysis and Synthesis with Applications to Communications and Signal/Image Processing, Kluwer Academic, pp. 17-22, 1997 


\section{Appendix}

\section{A. Proof of Lemma 5}

Let $Z \in G F(2)^{k}$ be a random variable independent of $X$ with uniform distribution. We have

$$
\begin{aligned}
& \sum_{w} \Delta(f(X, Z) \oplus w \cdot Z) \cdot \Delta(w \cdot g(Y) \oplus v \cdot Y) \\
& =\sum_{w} \mathrm{E}\left[(-1)^{f(X, Z) \oplus w \cdot Z}\right] \cdot \mathrm{E}\left[(-1)^{w \cdot g(Y) \oplus v \cdot Y}\right] \\
& =\sum_{w, x, y, z} \operatorname{Pr}(x, z) \cdot \operatorname{Pr}(y) \cdot(-1)^{f(x, z) \oplus v \cdot y \oplus w \cdot(z \oplus g(y))} \\
& =2^{k} \cdot \sum_{x, y} \operatorname{Pr}(X=x, Z=g(y)) \cdot \operatorname{Pr}(Y=y) \cdot(-1)^{f(x, g(y)) \oplus v \cdot y} \\
& =\sum_{x, y} \operatorname{Pr}(x, y) \cdot(-1)^{f(x, g(y)) \oplus v \cdot y} \\
& =\mathrm{E}\left[(-1)^{f(X, g(Y)) \oplus v \cdot Y}\right],
\end{aligned}
$$

which is $\Delta(f(X, g(Y)) \oplus v \cdot Y)$.

\section{B. Examples of Multiple Polynomials $Q(x)$}

Example of $\boldsymbol{Q}(\boldsymbol{x})$ with Low Degree. Here is a multiple polynomial of degree less than 855 with weight 31 :

$$
\begin{aligned}
Q_{1}(x)= & x^{668}+x^{579}+x^{553}+x^{313}+x^{262}+x^{121}+x^{117}+x^{109}+x^{106}+x^{101}+ \\
& x^{100}+x^{97}+x^{94}+x^{87}+x^{82}+x^{76}+x^{72}+x^{71}+x^{57}+x^{47}+ \\
& x^{40}+x^{37}+x^{34}+x^{32}+x^{23}+x^{21}+x^{17}+x^{16}+x^{3}+x^{2}+1 .
\end{aligned}
$$

Observe that $Q_{1}(x)$ is not optimal as $w_{855}=20$ from Table 3 .

Examples of $\boldsymbol{Q}(\boldsymbol{x})$ with Weight Four. Recall that $\theta_{i}=2^{L_{i}}-1$ is the order of $p_{i}(x)$ for $i=1,2,3,4$. By definition, $p_{i}(x) \mid x^{\theta_{i}}+1$. On the other hand, $p_{i}(x) p_{j}(x) \mid \operatorname{lcm}\left(x^{\theta_{i}}+1, x^{\theta_{j}}+1\right)=x^{\operatorname{lcm}\left(\theta_{i}, \theta_{j}\right)}+1$ for $i \neq j$, hence we deduce the following three multiple polynomials of $p(x)$ with weight 4 with ease:

$$
\begin{aligned}
& Q_{2}(x)=\left(x^{\operatorname{lcm}\left(\theta_{1}, \theta_{2}\right)}+1\right)\left(x^{\operatorname{lcm}\left(\theta_{3}, \theta_{4}\right)}+1\right), \\
& Q_{3}(x)=\left(x^{\operatorname{lcm}\left(\theta_{1}, \theta_{3}\right)}+1\right)\left(x^{\operatorname{lcm}\left(\theta_{2}, \theta_{4}\right)}+1\right), \\
& Q_{4}(x)=\left(x^{\operatorname{lcm}\left(\theta_{1}, \theta_{4}\right)}+1\right)\left(x^{\operatorname{lcm}\left(\theta_{2}, \theta_{3}\right)}+1\right),
\end{aligned}
$$

where

$$
\begin{aligned}
& \operatorname{lcm}\left(\theta_{1}, \theta_{2}\right)=2^{56}-2^{31}-2^{25}+1, \operatorname{lcm}\left(\theta_{1}, \theta_{3}\right)=2^{58}-2^{33}-2^{25}+1, \\
& \operatorname{lcm}\left(\theta_{1}, \theta_{4}\right)=2^{64}-2^{39}-2^{25}+1, \operatorname{lcm}\left(\theta_{2}, \theta_{3}\right)=2^{64}-2^{33}-2^{31}+1, \\
& \operatorname{lcm}\left(\theta_{2}, \theta_{4}\right)=2^{70}-2^{39}-2^{31}+1, \operatorname{lcm}\left(\theta_{3}, \theta_{4}\right)=\left(2^{39}-1\right) \sum_{k=0}^{10} 2^{3 k} .
\end{aligned}
$$

The degrees of $Q_{2}(x), Q_{3}(x), Q_{4}(x)$ are approximately $2^{69}, 2^{70}, 2^{65}$ respectively. Note that we may also expect optimal multiples with degree in the same order of magnitude and weight 3 . 
C. Table of $\log _{2}|\hat{\mathcal{D}}(a)|$ for $\ell=6$

Table 14. $\log _{2}\left|\hat{\mathcal{D}}\left(c_{t}^{0}, c_{t+1}^{0}, \ldots, c_{t+5}^{0}\right)\right|$ where dashed entries denote $-\infty$

\begin{tabular}{|c|c|c|c|c|c|c|c|c|c|}
\hline & \multicolumn{8}{|c|}{$c_{t+3}^{0} c_{t+4}^{0} c_{t+5}^{0}$} \\
\hline & & 000 & 001 & 010 & 011 & 100 & 101 & 110 & 111 \\
\hline \multirow{8}{*}{$c_{t}^{0} c_{t+1}^{0} c_{t+2}^{0}$} & 000 & 0 & - & - & - & - & - & - & - \\
\hline & 001 & - & - & - & -4 & - & - & - & - \\
\hline & $\overline{010}$ & - & - & - & - & - & - & -4 & - \\
\hline & 011 & - & - & - & - & - & -6 & - & -3.356 \\
\hline & 100 & - & -3.356 & - & - & - & - & - & -8 \\
\hline & 101 & - & - & - & - & -4 & - & - & - \\
\hline & 110 & - & -8 & - & -5.356 & - & -5.356 & - & -5.356 \\
\hline & 111 & - & - & -6 & - & - & - & -3.356 & - \\
\hline
\end{tabular}

